\title{
Study of phase change LIPPS formation in Si by fs-resolved microscopy
}

\author{
D. Puerto,* M. Garcia-Lechuga, J. Solis, and J. Siegel \\ Laser Processing Group, Instituto de Optica, CSIC, Serrano 121, 28006 Madrid, Spain \\ Authore-mail address: puerto@io.cfmac.csic.es
}

\begin{abstract}
We report the formation of amorphous-crystalline gratings in c-Si by fs laser irradiation at different wavelengths, repetition rates and angles of incidence. Using fs-resolved microscopy, we reveal the phase transformation dynamics of these structures.

OCIS codes: (050.6624) Subwavelength structures; (350.3390) Laser materials processing; (240.3695) Linear and nonlinear light scattering from surfaces; (320.7130) Ultrafast processes in condensed matter, including semiconductors.
\end{abstract}

\section{Introduction}

One of the most interesting properties of Silicon for the electronics industry is that it can be cycled between the crystalline and amorphous phase. The phase change can be induced by laser irradiation, with the amorphous phase being obtained by a melting process followed by fast quenching, while recrystallization is induced by laser thermal annealing. The use of ultrashort pulses (pico- and femtosecond) allows triggering non-thermal phase changes which may lead faster amorphization and recrystallization processes [1-2].

Another process that can be triggered in $\mathrm{Si}$ is the formation of Laser-Induced Periodic Surface Structures (LIPSS). This self-organization phenomenon is produced by an interference process between the incident laser light and a scattered surface wave, yielding topographic grating structures with an orientation typically perpendicular to the laser polarization and periods in the order of the laser wavelength [3]. While LIPPS have been observed mainly in the ablation regime [4], recently Katsumata et al. have observed self-organized periodic amorphous-crystalline structures [3] in thin GeTe films after irradiation with multiple femtosecond laser pulses [5].

\section{Result}

In this work, we combine both phenomena (laser-induced phase changes and LIPSS) to fabricate amorphous and crystalline grating structures. Our strategy for static irradiation is to use a first femtosecond laser pulse to form a homogeneous, ultrathin amorphous surface layer, whereas a second pulse triggers amorphous-crystalline LIPSS formation. The grating formed has a lateral extension of a few micrometers and a period close to the laser wavelength, which can be resolved by optical microscopy due to the different optical properties of the two structural phases of Si.
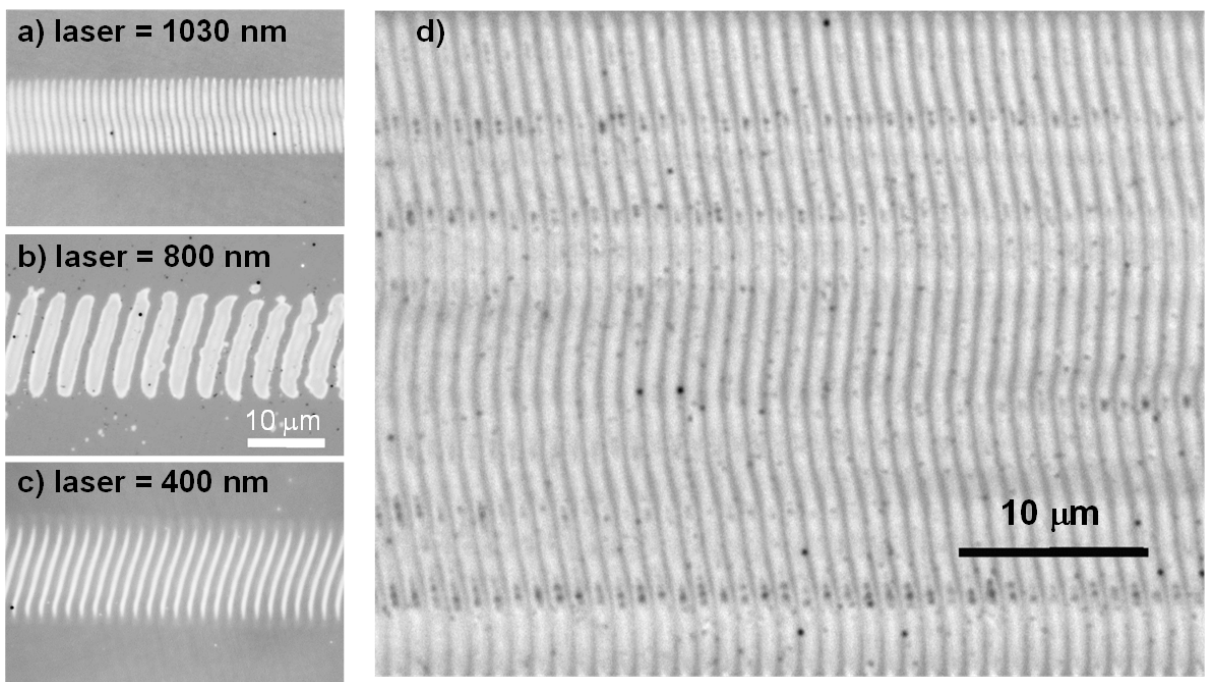

Figure 1. Optical micrographs of lines written under different laser (370 fs, $500 \mathrm{kHz}$ and $1030 \mathrm{~nm}$ (a), $100 \mathrm{fs}, 100 \mathrm{~Hz}, 800 \mathrm{~nm}$ (b) and $100 \mathrm{fs}$, $100 \mathrm{~Hz}, 400 \mathrm{~nm}(\mathrm{c}))$ and irradiation conditions (normal incidence (a), incident at an angle of $53^{\circ}(\mathrm{b}, \mathrm{c})$ ). The beam was horizontally polarized. (d) 2D amorphous-crystalline grating written under the same conditions as in (a). 
We have extended this static experiment into extended lines of gratings (Fig. 1a-c, Left) by scanning the laser beam over the surface. In order to identify the adequate spatial pulse overlap in scanning conditions we have written lines at different scan velocities. We found that a well-defined spatial pulse overlap needs to be kept in order to ensure the formation of pure amorphous-crystalline structures and that any deviation from it leads either to inhomogeneous or ablative structures. We also demonstrate that this irradiation strategy can be extended to other laser repetition rates and wavelengths (Fig. 1b-c, $100 \mathrm{~Hz}, 100 \mathrm{fs}, 800$ (b) and $400 \mathrm{~nm}(\mathrm{c})$ ), and that the grating period can be tuned by changing the angle of laser beam incidence (Fig. 1b-c, 53 ${ }^{\circ}$ ). Choosing a certain spacing between written lines allows extending these lines into large-area 2D structures by interconnecting ripples of each line (Fig. 1d, Right)

We will also present first results on the temporal and spatial dynamics of the ripple formation mechanism, obtained by means of a fs-resolved microscopy system. The time-resolved images (Fig. 2a) correspond to snapshots of the transient reflectivity of the sample surface in scanning conditions recorded at a certain delay time after the arrival of the pump pulse. The reflectivity profiles of these images (Fig. 2c-b) reveal that ultrafast melting and rapid solidification are involved in this type of LIPSS formation. In particular, our results are able to resolve the birth of a new ripple (Fig. 2c, "infinite" arrow), beginning with an ultrafast reflectivity increase, consistent with melting (Fig. 2b, $300 \mathrm{fs}$ ), and ending with rapid solidification after a few ns (Fig. 2c, $5 \mathrm{~ns}$ ). These findings shed new light on the ongoing vivid debate about the formation mechanism of LIPSS in Si, and the technique employed here might contribute to clarify the formation mechanism of other LIPSS types.
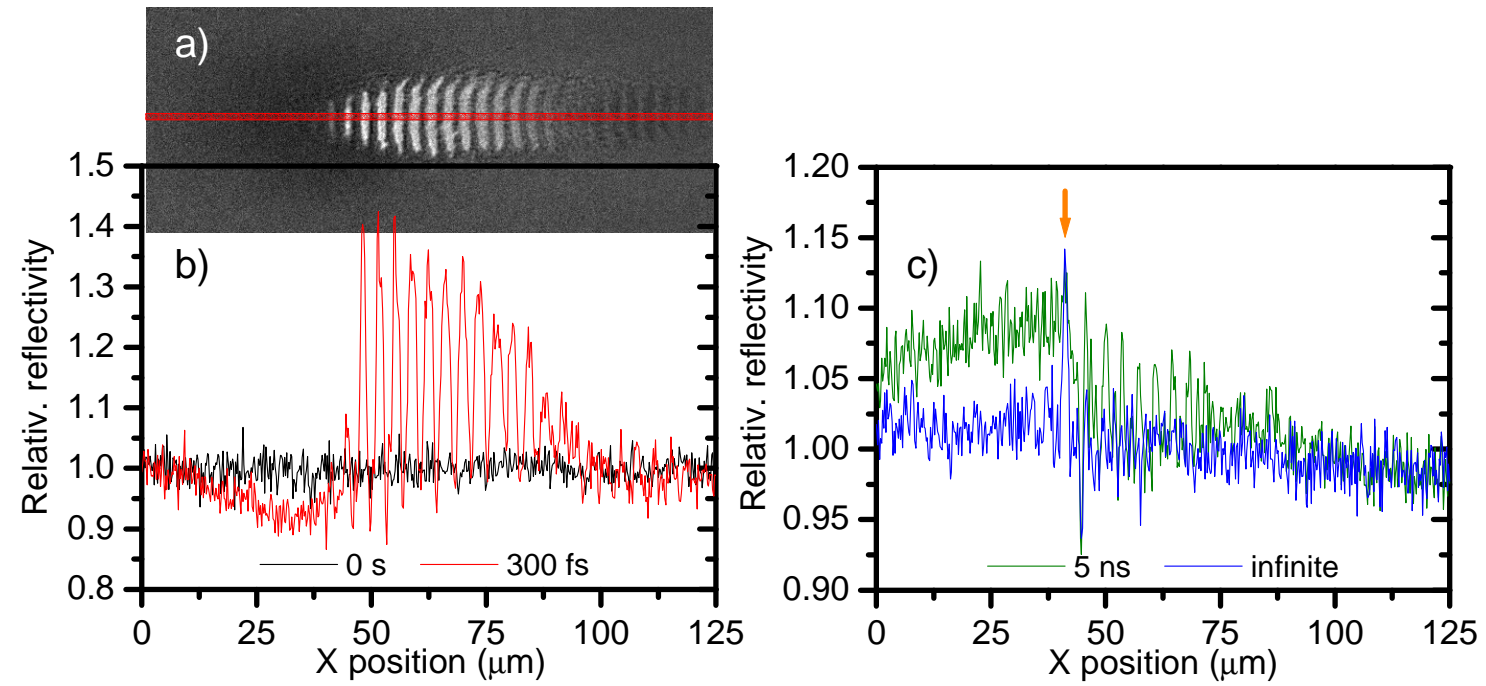

Figure 2. (a) Snapshot of the surface reflectivity $300 \mathrm{fs}$ after the arrival of the pump pulse $(800 \mathrm{~nm}, 100 \mathrm{fs}$, horizontally polarized, angle of incidence $53^{\circ}$ ) in writing conditions of a line of amorphous-crystalline gratings (b) Reflectivity profiles (b-c) of the irradiated region at different delays The arrow indicates the position of the new single ripple formed ("infinite").

\section{References}

[1] C.V. Shank, R. Yen, C. Hirlimann, "Time-Resolved Reflectivity Measurements of Femtosecond-Optical-Pulse-Induced Phase Transitions in Silicon,” Phys. Rev. Lett. 50, 454-457 (1983).

[2] Y. Izawa, Y. Izawa, Y. Setsuhara, M. Hashida, M. Fujita, R. S., H. Nagai, and M. Yoshida, "Ultrathin amorphous Si layer formation by femtosecond laser pulse Irradiation,” Appl. Phys. Lett. 90, 044107 (2007).

[3] J. F. Young, J. S. Preston, H. M. van Driel, and J. E. Sipe, "Laser-induced periodic surface structure. II. Experiments on Ge, Si, Al, and brass," Phys. Rev. B 27, 1155-1172 (1983).

[4] J. Bonse, S. Baudach, J. Krüger, W. Kautek, and M. Lenzner, "Femtosecond laser ablation of silicon-modification thresholds and morphology,” Appl. Phys. A: Mater. Sci. Process. 74, 19-25 (2002).

[5] Y. Katsumata, T. Morita, Y. Morimoto, T. Shintani, and T. Saiki, "Self-organization of a periodic structure between amorphous and crystalline phases in a GeTe thin film induced by femtosecond laser pulse amorphization,” Appl. Phys. Lett. 105, 031907 (2014). 